\title{
Tentativa y dolo eventual: una crítica de la jurisprudencia reciente de la Corte Suprema chilena
}

\author{
Criminal Attempts and dolus eventualis: \\ A Critique of some Recent Decisions by the \\ Chilean Supreme Court \\ Juan Pablo Mañalich R. ${ }^{*}$ \\ Alejandra Olave A.*
}

Recepción: 07/05/2021

Evaluación: 08/07/2021

Aceptación final: 30/08/2021

Resumen: Se somete a crítica una tendencia jurisprudencial de la Corte Suprema chilena, manifestada en una muy reciente sentencia. Se revisa el posicionamiento de la corte en lo concerniente a la naturaleza del dolo y al problema de su comprobación procesal, para después examinar su afirmación de la supuesta incompatibilidad entre tentativa y dolo eventual. En cuanto al primer punto, se destacan las deficiencias que exhibe la concepción psicologicista del dolo adoptada por la corte, contrastándola con una concepción débilmente adscriptivista. En lo tocante al segundo punto, se muestra cómo el argumento que la corte aduce para sostener la insuficiencia del dolo eventual para sustentar una responsabilidad por tentativa descansa en una errada interpretación del inc. $3^{\circ}$ del art. $7^{\circ}$ del Código Penal chileno.

Palabras clave: tentativa, dolo, Corte Suprema de Chile.

Doctor en derecho, Universidad de Bonn; profesor titular, Departamento de Ciencias Penales, Facultad de Derecho, Universidad de Chile, Santiago, Chile. Correo electrónico: jpmanalich@derecho.uchile.cl

** Magíster en derecho público, Universidad de Chile; profesora invitada, Área de Ciencias del Derecho, Facultad de Derecho, Universidad Nacional Andrés Bello, Santiago, Chile. Correo electrónico: aleolave@uchile.cl 
Juan Pablo Mañalich R., Alejandra Olave A.

\begin{abstract}
We criticize a noticeable line of precedent in the ruling of the Chilean Supreme Court, manifested in a recent decision. The court's stance concerning both the nature of the mens rea form known as dolus and the problem of its judicial proof is considered before we address its claim of the alleged incompatibility between the structure of criminal attempt and dolus eventualis. Regarding the former issue, the drawbacks of the court's psychologistic understanding of dolus are highlighted, for which we contrast it with a weakly ascriptivistic conception. Regarding the latter point, it is shown that the argument presented by the court for asserting the insufficiency of dolus eventualis as responsibility criterion for an attempted offense rests upon an incorrect reading of article 7.3 of the Chilean Penal Code.
\end{abstract}

Keywords: criminal attempts, dolus, Chilean Supreme Court.

\title{
1. La sentencia y su contexto
}

El 10 de noviembre de 2019, en el marco de manifestaciones y protestas que se extendían a lo largo del país con ocasión de lo que la prensa dio en llamar el "estallido social" chileno, el ciudadano estadounidense J.M.C. disparó, en múltiples ocasiones, un arma de fuego apuntando a un grupo de manifestantes en Viña del Mar. Poco antes de que hubiera transcurrido un año, el Tribunal Oral en lo Penal de Viña del Mar condenó al acusado como autor de un homicidio frustrado, de un homicidio tentado, así como de un delito (consumado) de disparo injustificado de un arma de fuego, imponiéndole una pena de cinco años y un día de presidio mayor en su grado mínimo por el primer hecho punible, y sendas penas de tres años y un día de presidio menor en su grado máximo por los dos últimos. ${ }^{1}$

1 De acuerdo con lo establecido en los arts. 51 y 52 del Código Penal chileno, la pena susceptible de ser impuesta sobre el autor de un crimen o simple delito frustrado se obtiene rebajando en un grado la pena prevista para el autor del respectivo hecho consumado. A su vez, la pena susceptible de ser impuesta sobre el autor de un crimen o simple delito (meramente) tentado se obtiene rebajando en dos grados la pena prevista para el autor del respectivo hecho consumado; en ambos casos, la rebaja en cuestión ha de practicarse a partir del umbral inferior de la pena legalmente prevista para el crimen o simple delito consumado. Ya que el marco penal asociado a un homicidio simple es de presidio mayor 
Tentativa y dolo eventual: una crítica de la jurisprudencia reciente...

Conociendo del recurso de nulidad interpuesto por la defensa del condenado, por sentencia de 17 de febrero de 2021 (rol No 134189-2020) la Corte Suprema (en adelante, también: "la corte") lo acogió parcialmente por decisión de mayoría, anulando la sentencia pronunciada por el tribunal de Viña del Mar en lo tocante a las primeras dos condenas. A través de la correspondiente sentencia de reemplazo, la corte condenó a J.M.C. como autor de los delitos consumados de lesión corporal simplemente grave y de daño simple, manteniendo además la condena por el delito de disparo injustificado de un arma de fuego.

Tal como se lee en el considerando $2^{\circ}$ de la sentencia de nulidad, $y$ para lo que aquí interesa, las circunstancias de hecho que el tribunal había declarado probadas, y que la corte tuvo por establecidas, son las siguientes:

El día 10 de noviembre de 2019, siendo alrededor de las 17:00 horas, se desarrollaba una manifestación pública masiva, en el sector céntrico de Reñaca, en Viña del Mar. En ese contexto, en la intersección de avenidas Ignacio Carrera Pinto y Borgoño, un grupo de manifestantes realizó bloqueos parciales de la calzada, controlando el paso de los vehículos a condición de que una persona (una por cada móvil) previamente descendiera a efectuar bailes o movimientos físicos. Hasta allí llegó [J.M.C.], a bordo de una camioneta [...], la que conducía. El grupo ubicado en el lugar intentó detener su móvil, lo que el acusado evitó acelerando el motor. Sin embargo, unos metros más allá de la referida esquina, por la calle Ignacio Carrera Pinto, se detuvo, extrajo una pistola marca Sig Sauer, Serie No40806, calibre .40 , inscrita a su nombre, sin autorización de porte, con la puerta del conductor del vehículo entreabierta y sin bajarse de la cabina, disparó hacia un grupo de manifestantes, impactando a [L.V.A.], provocándole una herida por proyectil de arma de fuego en muslo izquierdo de carácter grave, que tarda en sanar dos a tres meses con similar tiempo de incapacidad laboral.

en su grado medio (esto es, presidio de diez años y un día a quince años), la rebaja en un grado correspondiente a un homicidio frustrado lleva a una pena de presidio mayor en su grado mínimo (esto es, de cinco años y un día a diez años), mientras que la rebaja en dos grados correspondiente a un homicidio tentado lleva a una pena de presidio menor en su grado máximo (esto es, de tres años y un día a cinco años). 
Juan Pablo Mañalich R., Alejandra Olave A.

Hecho lo anterior, [J.M.C.] reanudó la marcha del móvil por la misma vía, para volver a detenerse a aproximadamente cien metros más allá, esta vez descendiendo del vehículo, para realizar dos disparos, uno de ellos dirigido al vehículo [...] conducido por [D.M.A.] y en el que también viajaba [N.P.C.], impacto que alcanzó el móvil señalado en su parte delantera izquierda, resultando ambos ocupantes ilesos. ${ }^{2}$

De las causales de nulidad invocadas por la defensa del condenado, aquella que la corte reconoció como base para acoger parcialmente el recurso es la prevista en la letra b) del art. 373 del Código Procesal Penal chileno (en adelante: "CPP”), consistente en que "en el pronunciamiento de la sentencia, se hubiere hecho una errónea aplicación del derecho que hubiere influido sustancialmente en lo dispositivo del fallo". En efecto, la corte validó el argumento esgrimido por el recurrente, según quien los "elementos fácticos" tenidos por probados por el tribunal, ${ }^{3}$ aunque en contra de lo sostenido por este, solo harían posible afirmar que J.M.C. actuó con dolo eventual de homicidio. Esto sería insuficiente para sustentar una condena por tentativa o por delito frustrado, puesto que una y otra forma de punibilidad solo serían compatibles con el dolo directo.

La corte insistió, así, en un posicionamiento que ella ha asumido en múltiples fallos anteriores. Ese posicionamiento concierne tanto a la cuestión de qué habría que entender por "dolo" y de cómo se comprueba si se satisfacen sus presupuestos, por un lado, como a la pretendida insuficiencia del dolo eventual para sustentar una imputación por tentativa o por delito frustrado, por otro. El presente comentario está destinado a examinar los argumentos presentados por la corte en lo tocante a cada uno de estos dos puntos. ${ }^{4}$

2 Cursivas suprimidas.

3 A saber: el hecho de haberse utilizado un arma de fuego, así como una munición de gran capacidad letal; el hecho de que el acusado haya disparado apuntando hacia una masa densa de personas; el hecho de que se haya tratado de disparos a corta distancia; el hecho de que, al efectuar los disparos, el acusado haya mostrado una actitud desafiante; y el hecho de que le haya sido imposible controlar el curso causal lesivo que desatara con sus disparos (considerando $15^{\circ}$ ).

4 No será considerada, en cambio, la calificación del segundo de los homicidios no consumados por los cuales fuera condenado el acusado como (meramente) tentado, y no frustrado, en razón de que esa calificación no llegó a ser, como tal, problematizada por la corte. El punto 
Tentativa y dolo eventual: una crítica de la jurisprudencia reciente...

Para ello, y con el propósito de simplificar la terminología, ocasionalmente usaremos la expresión "tentativa" en un sentido amplio, para designar tanto lo que el Código Penal chileno (en adelante: "CP”) denomina "tentativa" (stricto sensu) como lo que el mismo código llama "delito frustrado". Esto descansa en que un delito frustrado, tal como esta noción se encuentra definida en el inc. $2^{\circ}$ del art. $7^{\circ}$ del CP, se corresponde con una tentativa acabada (y no voluntariamente desistida), en tanto que lo que el inc. $3^{\circ}$ del mismo artículo define como "tentativa" se corresponde con una tentativa inacabada (Mañalich, 2017a, pp. 176 ss.; Olave, 2018, p. 176 , nota 2).

\section{El dolo y su comprobación procesal}

Sobre el primer punto, en el considerando $16^{\circ}$ de su sentencia la corte mantuvo, en términos literalmente coincidentes con lo declarado en algunos de sus fallos anteriores, que

la prueba del dolo - en cuanto se lo concibe como 'un conglomerado de hechos internos' - es una de las cuestiones más problemáticas en la sede procesal penal, ya que su acreditación en un caso concreto pasa por la necesidad de que se averigüen determinados datos de naturaleza psicológica: se debe averiguar una realidad que, como afirma expresivamente Herzberg, "se encuentra en la cabeza del autor" o, como puntualiza Schewe, se basa en "vivencias subjetivas del autor en el momento del hecho", unos fenómenos a los que puede y debe accederse en el momento posterior del proceso (Ragués i Vallés, 2002, p. 190).

es digno de mención, empero, en consideración a que en la segunda secuencia de disparos efectuados por el acusado pudieran haberse visto satisfechas, ceteris paribus, las condiciones para reconocer una tentativa acabada de homicidio, y así un homicidio frustrado. 
Juan Pablo Mañalich R., Alejandra Olave A.

Según la corte, de la "naturaleza psicológica" del dolo y su localización "en la cabeza del autor" (van Weezel, 2020, pp. 48 ss.) se seguiría que su comprobación procesal solo podría tener lugar a través de indicios, que hagan posible inferir su "presencia" a partir de la conducta del autor. Así, y tal como también se lee en el considerando $16^{\circ}$,

[e]l medio probatorio por excelencia al que se recurre en la praxis para determinar la concurrencia de los procesos psíquicos sobre los que se asienta el dolo no son ni las ciencias empíricas, ni la confesión auto inculpatoria del imputado, sino la llamada prueba indiciaria o circunstancial, plasmada en los denominados "juicios de inferencia" (Ragués i Vallés, cit., p. 238). Por su parte, el tratadista Pérez del Valle afirma que "la prueba de la concurrencia en un delito de los elementos subjetivos necesarios para imponer una sanción se desenvuelve en la jurisprudencia en un ámbito necesariamente vinculado a la prueba indiciaria, ya que el objeto de la convicción del tribunal es un elemento que, en principio, permanece reservado al individuo en el que se produce, de modo que para su averiguación o para su confirmación, se requiere una inferencia a partir de datos exteriores" (RDP, 1994, p. 413).

Además de concebir el dolo como un "conglomerado de hechos internos", y no obstante la manifiesta impertinencia de pretender apoyarse en Ragués para sustentarla, ${ }^{5}$ la corte ha sido relativamente consistente en defender una concepción dualista de aquel (Hernández 2011, pp. 71 ss.), identificándolo con la combinación de un elemento "cognitivo", constituido por la representación de las circunstancias con la que necesitaría contar el agente, ${ }^{6}$ y otro "volitivo", consistente en una actitud emocional referida al estado de cosas de cuya configuración dependa la consumación del delito.

5 Quien defiende una concepción cognitivista, en cuanto prescinde de la postulación de un componente volitivo o emocional, a la vez que adscriptivista del dolo, cuya "comprobación" dependería de la aplicación de un conjunto de "reglas sociales de atribución de conocimiento" (Ragués, 1999, pp. 357 ss., 362 ss.).

6 Para una problematización del recurso a la noción de conocimiento en la definición general del concepto de dolo, en atención a sus implicaciones semánticas y epistémicas, véase Mañalich (2020, pp. 24 ss., 29 ss.), donde se favorece una reconstrucción (monista) del dolo 
Tentativa y dolo eventual: una crítica de la jurisprudencia reciente...

Y una específica modalidad de este segundo componente "volitivo" - o más propiamente: emocional- marcaría, a su vez, la diferencia entre el dolo eventual y la llamada "imprudencia consciente" (Hernández, 2011, pp. 69 ss.). En efecto, y de acuerdo con lo afirmado por la corte en su emblemático fallo del 2 de julio de 2009 (rol No 3970-2008), ${ }^{7}$ el dolo eventual mostraría un plus subjetivo consistente en

la conformidad con [el] resultado, su aceptación o aprobación, dada a conocer con la continuación de la conducta peligrosa puesta en marcha, que pudo haber detenido; en otras palabras y como lo señalara Frank en su conocida segunda fórmula, el sujeto se dice a sí mismo, sea así o de otra manera, suceda esto o lo otro, en todo caso actúo; por ende, quien obra con dolo eventual renuncia a su posibilidad de detenerse en el curso desplegado y evitar de este modo la previsible y prevista lesión del objeto jurídico puesto en peligro; al agente no le importan las consecuencias lesivas de su proceder, aceptando que sobrevengan [considerando $14^{\circ}$ ].

Una particular implicación de semejante concepción a la vez psicologicista y dualista del dolo aparece en otra sentencia pronunciada por la Corte Suprema, del 10 de diciembre de 2020 (rol No 119315-2020). Aquí la corte anuló la sentencia del Tribunal de Juicio Oral en lo Penal de Colina, que había recalificado el hecho atribuido al imputado - consistente en haber efectuado un número indeterminado de disparos de bala contra un automóvil ocupado por tres personas-, preliminarmente calificado como constitutivo de un delito de daño, como constitutivo de un homicidio frustrado. En su invalidación de la sentencia del tribunal de Colina, la corte

como título (ordinario) de imputación subjetiva fundado en la adscripción de una actitud doxástica consistente en una creencia predictiva.

7 Se trata de la sentencia que confirmó la sentencia de la Corte de Apelaciones de Santiago que condenara, a título de homicidio imprudente, a un guardador y a una educadora de párvulos que se desempeñaban en una "sala cuna", por haber dado lugar a la muerte de un sietemesino por asfixia resultante de una sofocación, al haber el primero cubierto la boca del menor con una cinta adhesiva con la leyenda "soy un llorón", encontrándose este al cuidado de la segunda, quien sin remover la cinta posteriormente lo acostó en una cuna. Acerca del fallo de la Corte Suprema, véase Krause (2015); también van Weezel (2011, pp. 44 ss.; 2020, pp. 43 ss.). 
Juan Pablo Mañalich R., Alejandra Olave A.

afirmó que la recalificación efectuada por el tribunal habría traído aparejada una modificación de los hechos fijados en la acusación, lo cual contravendría lo establecido en el art. 341 del CPP, que consagra el principio de congruencia qua exigencia de identidad factual entre el objeto temático de la acusación y el de la sentencia condenatoria (considerando 14\%).

Junto con afirmar que el dolo directo sería "el ingrediente subjetivo indispensable para la configuración de un delito frustrado", la corte observó que la decisión de tener por comprobada la existencia de un dolo directo de homicidio implicaría la adición de "un hecho nuevo y sorpresivo para la defensa, atada [esta] a la descripción fáctica de la acusación" (considerando $14^{\circ}$ ), consistente ese hecho nuevo en el pretendido "ingrediente subjetivo" que sería codefinitorio de la noción de dolo directo. Esto vuelve manifiesto que la denuncia de una vulneración del principio de congruencia solo es inteligible si se postula, como lo hiciera la corte, que la comprobación procesal del dolo directo dependería del establecimiento de hechos diferentes de aquellos en cuyo establecimiento pudiera descansar una comprobación de dolo eventual.

Esto último es algo que necesita ser al menos matizado si se advierte que una concepción psicologicista (de la comprobación) del dolo, como la favorecida por la corte, presenta deficiencias suficientemente serias como para que se justifique su reemplazo por una concepción que cabría caracterizar como débilmente adscriptivista (de la fundamentación) del dolo. ${ }^{8}$ Esas deficiencias se expresan en que, bajo una concepción psicologicista, la fundamentación del dolo devenga en argumentativamente incontrolable (Ragués, 1999, pp. 259 ss., 271 ss.). Esto vuelve preferible un método de fundamentación del dolo que descanse en la aplicación de un conjunto de indicadores-de-dolo, consistentes en descriptores de "síndromes de

8 El carácter débilmente adscriptivista de la concepción aquí esbozada se expresa en que, bajo esta, el dolo no es identificado con un "juicio de imputación", sino más bien con un título de imputación que descansa en la adscripción de una creencia predictiva referida a la ejemplificación, por parte del comportamiento potencialmente imputable como delictivo, de las propiedades sobre las cuales tendría que supervenir su antinormatividad en cuanto instancia del respectivo género delictivo; al respecto, Mañalich (2020, pp. 18 ss., 31 ss.). En estos términos, el carácter (débilmente) adscriptivo de la operación de fundamentación del dolo no responde al hecho de que, en cuanto título de imputación, el dolo sea inde- 
Tentativa y dolo eventual: una crítica de la jurisprudencia reciente...

riesgo" específicamente referidos a la realización del respectivo tipo-dedelito. Puesto que, a diferencia de un indicio, cuya relación con aquello de lo cual es indiciario es de naturaleza empírica, un indicador se encuentra conceptualmente conectado con aquello de lo cual es indicativo, la satisfacción del respectivo catálogo de indicadores es aquello en lo que consiste una adscripción de dolo, y no algo que deba ser ulteriormente valorado en la aplicación del concepto de dolo. ${ }^{9}$

Lo anterior se traduce en que la base fáctica de una posible adscripción de dolo quede reducida al conjunto de circunstancias cuya comprobación habilitaría al tribunal competente para tener por satisfechos uno o más indicadores-de-dolo. Con ello, la plausibilidad de la sugerencia de que, para el pronunciamiento de una condena por un hecho punible subjetivamente imputable a título de dolo directo, sería insuficiente una base fáctica a su vez (mínimamente) suficiente para sustentar una condena fundada en una adscripción de dolo eventual dependería, críticamente, de que tenga sentido reconocer un catálogo diferenciado de indicadores de dolo directo, en cuanto forma de dolo "más exigente". Y que esto último tenga sentido depende, a su vez, de que haya razones para diferenciar varias formas de dolo, lo cual tendría que depender de que la respectiva regulación legal imponga, o vuelva necesaria, semejante diferenciación. ${ }^{10}$

pendiente de, o insensible a, los estados mentales atribuibles al agente; se trata, antes bien, de que la creencia predictiva que, en cuanto actitud proposicional, ha de ser atribuible al potencial autor para que el hecho en cuestión le sea imputable a título de dolo es algo cuya constatación no es conceptualmente diferenciable de su adscripción, tal como ello es predicable, en general, de cualquier actitud proposicional. Con esto, la premisa determinante para advertir el carácter adscriptivo de la operación de fundamentación del dolo se obtiene de consideraciones propias de la filosofía de la mente, y no de consideraciones (inmediatamente) pertenecientes a la dogmática del dolo. Para una revisión contrastiva de las diferentes versiones de una concepción adscriptivista de la fundamentación del dolo reconocibles en la literatura chilena más reciente, véase van Weezel (2021, pp. 192 ss.).

9 En este punto radica el aspecto crucialmente diferenciador del planteamiento aquí favorecido frente al modelo de "tres peldaños" presentado por Londoño (2020, pp. 422 ss.). Bajo el modelo aquí adoptado, no cabe reconocer más que dos "peldaños": el de la comprobación de las circunstancias en referencia a las cuales pudieran ser aplicados los correspondientes indicadores-de-dolo, que es quaestio facti, y el de la fundamentación de la adscripción del dolo como resultado de la aplicación de los indicadores, que es quaestio iuris.

${ }^{10} \mathrm{Al}$ respecto, y críticamente, van Weezel (2011, pp. 25 ss., 34 ss., 51). 
Juan Pablo Mañalich R., Alejandra Olave A.

La demostración de que, contra lo sostenido por la Corte Suprema, el $\mathrm{CP}$ no sujeta la punibilidad de la tentativa (lato sensu) a la satisfacción de una forma de dolo más exigente que aquella que necesita ser satisfecha para que resulte punible el respectivo delito consumado podría, así, contribuir al desarrollo de una práctica jurisprudencial que uniforme los presupuestos de la imputación subjetiva de cuya satisfacción dependería, "por defecto", una decisión de condena, identificando esos presupuestos con las condiciones de adscripción de dolo eventual (Mañalich, 2020, pp. 14 ss.).

\section{3. ¿Incompatibilidad entre tentativa y dolo eventual?}

En la sentencia a cuyo análisis se destina el presente comentario, la corte no llegó a verse enfrentada al problema recién reseñado. Esto, porque la decisión del voto de mayoría pasó, en lo fundamental, por simplemente negar que concurriera el "ingrediente subjetivo" que sería distintivo del dolo directo, en consideración a que el condenado habría actuado únicamente con dolo eventual, siendo esta última forma de dolo insuficiente para una imputación a título de tentativa. Tal como ya se anticipara, esta pretendida insuficiencia del dolo eventual para sustentar una imputación a título de tentativa da forma a una línea de decisión claramente reconocible en la jurisprudencia de la corte. Esta queda ejemplificada, entre otras, en su muy polémica sentencia de 11 de julio de 2017 (rol № 19008-2017), referida a un caso que involucraba una muestra especialmente cruenta de violencia machista. En lo que aquí interesa, la corte desechó la calificación de uno de los hechos atribuidos al condenado como constitutivo de un femicidio frustrado, en razón de la supuesta incompatibilidad que se daría entre esta forma de punibilidad y el dolo eventual (Mañalich, 2017a; Londoño, 2020, pp. 418 ss.).

En congruencia con lo anterior, y pretendiendo contar con el apoyo de "sólidas opiniones doctrinarias", ${ }^{11}$ la corte dedicó los considerandos $18^{\circ} \mathrm{y}$

${ }^{11}$ A propósito de lo cual la corte menciona a Novoa y a Garrido como las únicas voces discordantes reconocibles en la discusión doctrinal chilena a este respecto. Ello es acertadamente refutado en el considerando $6^{\circ}$ del voto disidente, donde la nómina de autores proclives a reconocer - sea genéricamente, sea en relación con determinadas especies de comportamiento punible - la compatibilidad entre tentativa (lato sensu) y dolo eventual es 
Tentativa y dolo eventual: una crítica de la jurisprudencia reciente...

siguientes de la sentencia ahora analizada a sustentar, una vez más, la tesis de la incompatibilidad, la cual se resumiría en que

tanto el delito frustrado como la tentativa $[. .$.$] requieren dolo$ directo en el agente, ya que la etapa de frustración del iter criminis no se diferencia en nada - en el plano subjetivo- de la tentativa, fase que, al exigir hechos directamente encaminados a la consumación, sólo [sic] se realiza con dolo directo, esto es, con intención o propósito de lograr la consumación del ilícito, mismo requisito que debe concurrir en la frustración [considerando $18^{\circ}$ ].

De este modo, la corte fundamentó la pretendida exigencia de dolo directo en lo establecido en el art. $7^{\circ}$ inc. $3^{\circ}$ del CP, que condiciona la existencia de una tentativa inacabada a que el agente dé "principio a la ejecución del crimen o simple delito por hechos directos”. Así, y en la medida en que "en cuanto al dolo de la frustración, no hay diferencia con el dolo de la tentativa, entonces, aquél se integra con una voluntad dirigida a alcanzar la comisión total, plena, del tipo penal, esto es, dolo directo de consumar la lesión del objeto jurídico protegido" (considerando 18º). De ahí que la corte concluyera

[q]ue de la lectura del motivo décimo cuarto del fallo en alzada es factible desprender que los argumentos tenidos en consideración por los sentenciadores del grado para establecer la concurrencia del dolo directo en el obrar del acusado -esto es, que el hechor no sólo se representó el resultado de muerte al disparar en contra de los ofendidos, sino que además quiso su materialización-, son contradictorios con tal hipótesis, toda vez los mismos dan cuenta de aseveraciones que son propias de una conducta diversa de aquella que se pretendió configurar [considerando $\left.21^{\circ}\right]_{.}^{12}$

enriquecida con referencias a Politoff, Náquira y Vargas Viancos. Las más recientes tomas de posición ofrecidas en Londoño (2016), Schürmann (2016), y Mañalich (2017a), llevan a que el conjunto en cuestión sea aún más vasto.

${ }^{12}$ Cursivas en el original. 
Juan Pablo Mañalich R., Alejandra Olave A.

Puesto que el análisis de cada uno de los "elementos fácticos" en los cuales el tribunal a quo había pretendido sustentar la comprobación de un dolo directo de homicidio solo haría posible, según la corte, tener por comprobado un dolo eventual de homicidio (véase el considerando $22^{\circ}$ ), ello bastaría para desestimar una condena por homicidio frustrado o tentado.

El problema está, sin embargo, en que el razonamiento de la corte descansa en una muy sutil maniobra interpretativa que solo puede ser caracterizada como falaz. Pues tal como queda de manifiesto en el pasaje ya reproducido del considerando $18^{\circ}$ de su sentencia, la corte nuevamente se permitió parafrasear los términos con los cuales el inc. $3^{\circ}$ del art. $7^{\circ} \mathrm{del} \mathrm{CP}$ define el concepto legal de tentativa (stricto sensu), de un modo que resulta en una tergiversación de esa misma definición. En efecto, mientras que la disposición legal exige que el agente dé principio a la ejecución del delito "por hechos directos", la corte argumenta como si esto fuera equivalente a una exigencia de "hechos directamente encaminados a la consumación".

Con ello, la corte pasa enteramente por alto que la interpretación más plausible de la expresión "por hechos directos" consiste en atribuir a esta cláusula la función de demarcar el inicio de una tentativa frente al ámbito de la llamada "preparación” (Mañalich, 2021, secc. 1). Más precisamente: la exigencia de que el principio de ejecución del delito en cuestión tenga lugar a través de "hechos directos" admite ser tomada como una exigencia de inmediatez-de-acción, en el sentido de que solo puede reconocerse como iniciada una tentativa si el agente, de acuerdo con su representación de las circunstancias, se encuentra en una situación en la que pueda ejecutar la acción que habría de realizar el tipo (Mañalich 2019a, pp. 828 ss., 832 ss.).

Si hubiera que mostrar caridad interpretativa hacia la tergiversación de la formulación legal del criterio de reconocimiento de un inicio de la tentativa (stricto sensu) en la que - literalmente - reincidió la Corte Suprema en el fallo aquí considerado (Mañalich, 2017a, p. 177), cabría reparar en que la estructura de una tentativa inacabada efectivamente vuelve necesario que al autor pueda atribuirse una intención distintiva, a saber: la intención de completar la ejecución de la acción en la que, objetivamente, habría de consistir el delito (Mañalich, 2019b, pp. 30 ss., 37 ss.). La necesidad de que 
Tentativa y dolo eventual: una crítica de la jurisprudencia reciente...

al agente pueda adscribirse esta intención distintiva, cuya denominación dogmática más extendida es la de "resolución-al-hecho", se sigue de la propia definición del concepto de tentativa (stricto sensu) plasmada en el inc. $3^{\circ}$ del art. $7^{\circ}$ : que de acuerdo con esta definición una tentativa, qua tentativa inacabada, se caracterice por la circunstancia de que, a pesar de haberse dado principio a la ejecución del delito "por hechos directos", para el condicionamiento de la consumación del delito hayan "falt[ado] uno o más [hechos asimismo directos] para su complemento", determina que la tematización de semejante tentativa del delito en cuestión solo sea inteligible con cargo a la hipótesis de que el agente se encontraba resuelto a completar esa ejecución. Y esta hipótesis se ve corroborada cuando resultan satisfechas las condiciones para atribuir la correspondiente resolución-al-hecho al potencial autor.

Lo anterior vuelve manifiesto que la corte yerra al pretender equiparar los presupuestos subjetivos de una tentativa (stricto sensu) y de un delito frustrado (considerando $28^{\circ}$ ). Pues que sea definitorio de un delito frustrado que el agente haya puesto "de su parte todo lo necesario para que el delito se consume" determina que, para que se satisfaga la definición del inc. $2^{\circ}$ del art. $7^{\circ}$ del CP, sea necesario que la ejecución de la acción que, según la representación que el agente haya tenido de las circunstancias, habría podido realizar el tipo haya de considerarse completada. En esto consiste el hecho de que un delito frustrado se corresponda con una tentativa acabada. De ahí que para la configuración de un delito frustrado no sea en absoluto necesario siquiera preguntar si al autor podía atribuirse una resolución-al-hecho, precisamente porque aquí el autor ha llegado a completar la ejecución de una acción que, según su representación de las circunstancias, tendría que haber sido suficiente para hacer relevantemente probable la consumación del delito. Tratándose de una tentativa acabada, la exigencia de una resolución-al-hecho resulta, por ello, enteramente superflua.

Pero aun restringiendo el foco a la tentativa inacabada, cuya estructura sí exige la actualización de una intención previa en la forma de una resolución-al-hecho, es un error extraer de esta exigencia la supuesta necesidad de un dolo directo. Para advertir por qué, considérese el caso siguiente (Mañalich, 2019b, pp. 45 ss.): 
Juan Pablo Mañalich R., Alejandra Olave A.

A pretende ejercitar su puntería en el arte del disparo con una escopeta, para lo cual selecciona un blanco ubicado a una distancia tal que para A resulta dudoso si se trata de un espantapájaros, como él quisiera que fuera, o en cambio de un trabajador agrícola que pudiera encontrarse de pie, contemplando el horizonte. Tras apuntar hacia su objetivo, y en el instante de empezar a mover su dedo índice para tirar del gatillo, un tercero, que tampoco sabe si aquello contra lo cual A está ad portas de disparar consiste en un espantapájaros o un ser humano vivo, se lanza sobre A, botándolo al suelo e impidiendo así que este logre disparar.

Es claro que, al disponerse a efectuar el disparo, A no tenía la intención - sea última o intermedia - de matar a otro ser humano, puesto que lo que él perseguía era, únicamente, que el proyectil por él disparado impactara en el "blanco". Esto tendría que bastar para descartar una adscripción de dolo de homicidio en la forma de un dolo directo de primer grado (o dolo "de propósito"). Al mismo tiempo, la circunstancia de que para A haya sido dudoso que aquello contra lo cual se disponía a disparar fuera o bien un espantapájaros o bien un ser humano (vivo) lleva a que también deba descartarse un dolo de homicidio en la forma de un dolo directo de segundo grado (o dolo "de las consecuencias necesarias"). Antes bien, a A solo cabe atribuir un dolo de homicidio en la forma de un dolo eventual, a pesar de que todo habla a favor de atribuirle, al mismo tiempo, la requerida resolución-al-hecho, constituida por la intención de disparar contra aquello que identificaba como el respectivo "blanco".

Que la coexistencia de esta resolución-al-hecho y de un dolo (solo) eventual de homicidio no debería dar lugar a perplejidad alguna, se sigue de que la descripción de lo que A estaba ad portas de hacer bajo la cual cabe atribuirle la intención en la que consistía la resolución-al-hecho no coincide con la descripción que confería potencial significación delictiva a ese mismo disparo, siendo esta última descripción la determinante para fundar la correspondiente adscripción de dolo. ${ }^{13}$ Bajo la descripción del inminente

${ }^{13}$ Esto se explica por el hecho de que los verbos y en general las expresiones lingüísticas de las cuales se vale quien adscribe actitudes proposicionales o en general "estados intencionales" - como lo son tanto una intención (stricto sensu) como una creencia- a alguien sean expresiones cuyo uso distintivamente genera contextos intensionales (en oposición 
Tentativa y dolo eventual: una crítica de la jurisprudencia reciente...

disparo como "disparar contra algo que es o bien espantapájaros o bien un ser humano (vivo)", A tiene la intención de efectuar el disparo, mas no bajo su descripción como "matar a un ser humano (vivo)". Esta última es una descripción cuya satisfacción a través del disparo que él se disponía a efectuar solo viene en consideración como el objeto de una creencia predictiva - no revestida de una "seguridad rayana en la certeza" - atribuible a A, en la medida en que, con base en el correspondiente catálogo de indicadores de dolo de homicidio, pueda decirse que, al empezar a tirar del gatillo del arma, A se representaba estar empezando a hacer algo a través de lo cual, en caso de lograr completar el disparo, él habría generado un concreto riesgo de muerte para otro ser humano.

Con ello, la tentativa inacabada de homicidio imputable a A se constituye a través de un comportamiento consistente en haber dado inicio a la realización de la intención de disparar un arma de fuego en una determinada dirección, a pesar de serle atribuible la creencia predictiva de que ello eventualmente habría de resultar en la muerte de otro ser humano. Esto muestra que, en contra de lo sostenido por la Corte Suprema, la estructura de la tentativa inacabada es compatible con el dolo eventual. Y ya contamos con una explicación suficientemente precisa de lo que subyace a su resistencia a reconocer esa compatibilidad, a saber: la confusión de la resolución-al-hecho en cuanto criterio de compensación del carácter inacabado de la tentativa en cuestión, por un lado, con el dolo en cuanto criterio de imputación subjetiva, por otro. No estaría de más observar que esa confusión tiene consecuencias más vastas que las que la corte se muestra dispuesta a asumir. Pues en la medida en que la supuesta exigencia de dolo directo sea identificada con la exigencia de una "intención o propósito de lograr la consumación del ilícito”, de ello tendría que seguirse que también quede des-

a extensionales), esto es, contextos en los cuales la verdad de la oración empleada en la adscripción de la respectiva actitud o estado intencional no se ve necesariamente preservada si la descripción de su objeto es reemplazada por una descripción coextensiva. A modo de ejemplo: que Edipo llegara a formarse la intención de matar al viajero con el cual se cruzara en su camino hacia Tebas, no implica que Edipo haya llegado a formarse la intención de matar a Layo, su padre, a pesar de que el viajero con el cual Edipo se cruzara en el camino era (idéntico a) Layo, su padre. 
cartada la posibilidad de una tentativa con dolo directo de segundo grado. ${ }^{14}$ Y esto vuelve todavía más implausible esa misma identificación.

La denuncia de este fundamental yerro argumentativo que exhibe la sentencia aquí comentada deja intacto, por cierto, el optimismo que suscita el voto de minoría suscrito por el ministro Brito y el abogado integrante Munita. En el ínterin, ese optimismo se ha visto afianzado por la sentencia que la corte pronunció, por decisión de mayoría, el pasado 5 de mayo de 2021 ( $r o l N^{\circ} 16.945-2021$ ) - apenas transcurrido un poco más de dos meses desde que fuera dictado el fallo aquí comentado-, y que en lo fundamental reprodujo el argumento presentado en el voto de minoría de Brito y Munita para sostener la compatibilidad entre tentativa (lato sensu) y dolo eventual. Con todo, el optimismo queda opacado por el hecho de que en ese todavía más reciente fallo de mayoría no haya sido siquiera tematizada la línea de decisión de la cual, por el momento, la corte

${ }^{14}$ A este respecto se suscita, con todo, una dificultad en la interpretación de lo sostenido por la corte. En el considerando $21^{\circ}$ de su sentencia, y con base en el elemento fáctico constituido por la "utilización del arma de fuego", ella afirmó que "el encartado debió a los menos representarse que el uso de un arma de fuego a corta distancia podía ocasionar la muerte del ofendido L.A.V., fundamento que por cierto es propio de un obrar con dolo indirecto". La dificultad surge en razón de que no es infrecuente que, en la discusión doctrinal chilena, por "dolo indirecto" se entienda el llamado "dolo directo de segundo grado"; véase Etcheberry (1997, p. 297); Garrido (2003, p. 79); Hernández (2011, p. 70). Sin embargo, la circunstancia de que la expresión en cuestión haya sido usada por la corte para designar una forma de dolo que se vería configurada por el hecho de que el acusado "debi[era] representarse" que su acción "podía ocasionar la muerte" de la víctima habla a favor, más bien, de tomarla en el sentido en que, más tradicionalmente, el dolus indirectus viene en consideración como la forma embrionaria de lo que hoy denominamos "dolo eventual". Sobre ello, véase solamente Puppe (2010, pp. 44 ss.), quien ofrece una descripción de la transición conceptual que lleva de la construcción del dolus indirectus al reconocimiento del dolus eventualis. Esta hipótesis interpretativa se ve decisivamente corroborada por el hecho de que, en el contexto del mismo considerando $21^{\circ}$ de su sentencia, la corte observara que las disquisiciones con las cuales el tribunal había pretendido sustentar la afirmación de que el acusado "quiso" la materialización del resultado de muerte, con base en su experticia en el manejo de armas de fuego, funcionarían más bien como argumentos "que descartan la existencia de un dolo directo y reafirman la concurrencia en la especie de uno de carácter eventual”. Precisamente porque la corte parece reducir la contraposición relevante al contraste entre el dolo directo de primer grado y el dolo eventual, ella pasa por alto el lugar que, según su propio razonamiento, tendría que ocupar el dolo directo de segundo grado. 
Tentativa y dolo eventual: una crítica de la jurisprudencia reciente...

se ha distanciado a través de semejante drástico giro. Y esto parece volver aconsejable mantener algún grado de escepticismo en cuanto a la perdurabilidad de ese mismo giro.

En lo que ahora interesa, el precedente inmediato del voto de minoría con el cual Brito y Munita disintieron de la sentencia aquí analizada lo representa el excepcionalmente bien fundado voto que emitiera el entonces ministro Juica en su disidencia a lo resuelto por la corte mediante su sentencia, ya aludida, del 11 de julio de 2017 (Mañalich, 2017a, p. 178). El argumento por ellos esgrimido en defensa de la compatibilidad entre la forma de punibilidad de la tentativa lato sensu y el dolo eventual apunta, en efecto, al factor dogmáticamente crucial. Citando la autoridad de Max Ernst Mayer, el ministro Brito y el abogado integrante Munita sostuvieron que "el dolo de la tentativa es el mismo dolo de la consumación, como quiera que la tentativa no es un delito en sí mismo, sino forma imperfecta de un delito determinado, un tipo dependiente de otro autónomo, que yace en la Parte especial" [considerando $\left.5^{\circ}\right]^{15}$

Que esta es la consideración decisiva para rechazar la tesis asumida en el voto de mayoría, se sigue de que ella concierne a la relación en la que se encuentran la categoría de la consumación, por un lado, y las categorías de la frustración y la tentativa (stricto sensu), por otro.

Que un hecho punible cuente como consumado, como frustrado o como (meramente) tentado, solo es susceptible de ser constatado ex post. Esto, porque solo ex post es posible determinar si el comportamiento imputable al agente llega a satisfacer, o no, la descripción de cuya satisfacción dependería que ese comportamiento realice (antijurídicamente) el tipo de que se trate (Mañalich, 2017b, pp. 475 ss.). De ahí que solamente el autor del homicidio consumado pueda ser responsabilizado de haber producido la muerte de otro ser humano.

Lo anterior descansa en que la consumación de un homicidio no es otra cosa que la congruencia entre la descripción que termina siendo verdadera

${ }^{15}$ Que el pretendido "tipo dependiente" de la tentativa se corresponde, más bien, con un "esquema" de punibilidad dependiente, que como tal necesita ser puesto en relación con algún "tipo legal" que especifica una forma de comportamiento autónomamente dotada de significación jurídico-penal, lo advirtió perspicazmente Beling (1930). Al respecto, Mañalich (2017b, pp. 467 ss.). 
Juan Pablo Mañalich R., Alejandra Olave A.

del comportamiento imputable a su autor y la descripción que especifica el objeto de la creencia predictiva en virtud de la cual el mismo comportamiento le es imputable a título de dolo. De ahí que la consumación de un homicidio se corresponda con la realización del riesgo de muerte que el agente, sobre la base de su representación de las circunstancias, pudo decidir no generar, pero que decidió, no obstante, generar.

La falta de esa misma congruencia es definitoria, en cambio, de una tentativa (lato sensu) de homicidio. Pero tal como lo sugiere el voto de minoría, el autor de una tentativa de homicidio se comporta de un modo que hace reconocible exactamente la misma decisión que aquella que adopta el autor de un homicidio consumado. Y si la decisión de generar el correspondiente riesgo concreto de muerte para otro ser humano es idéntica en uno y otro caso, entonces es muy difícil explicar que un agente que alcanzó - tratándose de un homicidio frustrado- o comenzó - tratándose de un homicidio (meramente) tentado- a implementar esa decisión, no haya de ser responsabilizado por haberse comportado de un modo que, de haber resultado verdadera la creencia predictiva que le era atribuible, habría dado lugar a la muerte de otro ser humano.

\section{Bibliografía}

Beling, E. (1930). Die Lehre vom Tatbestand. Tübingen: J.C.B. Mohr, Paul Siebeck.

Etcheberry, A. (1997). Derecho Penal. Parte General, Tomo I (3a ed.). Santiago: Editorial Jurídica de Chile.

Garrido, M. (2003). Derecho Penal. Parte General, Tomo II (3a ed.). Santiago: Editorial Jurídica de Chile.

Hernández, H. (2011). Art. 1º. En J. Couso y H. Hernández (dirs.), Código Penal Comentado. Parte General (pp.10-105). Santiago: Abeledo Perrot y Legal Publishing.

Krause, M. S. (2015). Caso "cinta adhesiva" y la no aceptación de la Muerte. En T. Vargas (dir.), Casos Destacados de Derecho Penal. Parte General (pp. 105-112). Santiago: Thomson Reuters. 
Londoño, F. (2020). Tres peldaños para la prueba del dolo. Consideraciones a propósito de un polémico caso de femicidio frustrado. En A. Perin (ed.), Imputación penal y culpabilidad (pp. 413-436). Valencia: Tirant lo Blanch.

Londoño, F. (2016). Estudio sobre la punibilidad de la tentativa con dolo eventual en Chile. ¿Hacia una noción de tipo penal diferenciado para la tentativa? Revista de Ciencias Penales 93(3), 95-130. Disponible en: http://revistadecienciaspenales.cl/wp-content/uploads/2018/04/ Derecho-y-Ciencias-Penales-5.pdf

Mañalich, J. P. (2021). Principio de ejecución e inmediatez-de-acción. Revista de Derecho (Coquimbo. En línea), 28, e3480. Disponible en: https:// revistaderecho.ucn.cl/index.php/revista-derecho/article/view/3480

Mañalich, J. P. (2020). El dolo como creencia predictiva. Revista de Ciencias Penales LXVII (1er semestre), 13-42. Disponible en: http:// revistadecienciaspenales.cl/wp-content/uploads/2020/12/Revista-CPPrimer-Semestre-2020-corregida-21-49.pdf

Mañalich, J. P. (2019a). Inicio de la tentativa y oportunidad-para-la-acción. Revista Chilena de Derecho, 46(3), 821-844. Disponible en: http://ojs. uc.cl/index.php/Rchd/article/view/27143

Mañalich, J. P. (2019b). Tentativa y resolución-al-hecho. Isonomía, 51, 29-64. Disponible en: http://www.scielo.org.mx/pdf/is/n51/14050218-is-51-00029.pdf

Mañalich, J. P. (2017a). ¿Incompatibilidad entre frustración y dolo eventual? Revista de Estudios de la Justicia, 27, 171-182. Disponible en: https://rej. uchile.cl/index.php/RECEJ/article/view/47965

Mañalich, J. P. (2017b). La tentativa de delito como hecho punible. Revista Chilena de Derecho, 44(2), 461-493. Disponible en: http://ojs.uc.cl/ index.php/Rchd/article/view/4290/3996.

Olave, A. (2018). El delito de hurto como tipo de delito de resultado. Política Criminal, 13(25), 175-207. Disponible en: http://politcrim.com/ wp-content/uploads/2019/04/Vol13N25A5.pdf

Puppe, I. (2010). La distinción entre dolo e imprudencia (trad. de M. Sancinetti). Buenos Aires: Hammurabi.

Ragués, R. (1999). El dolo y su prueba en el proceso penal. Barcelona: J. M. Bosch. 
Juan Pablo Mañalich R., Alejandra Olave A.

Schürmann, M. (2016). Comentario de sentencia rol No 2512-2015 de la Ilustrísima Corte de Apelaciones de San Miguel: La punibilidad del homicidio frustrado cometido con dolo eventual. Revista de Ciencias Penales, XLIII(2), 273-282. Disponible en: http:// revistadecienciaspenales.cl/wp-content/uploads/2018/04/Corte-deApelaciones-5-1.pdf

van Weezel, Á. (2021). Intención, azar e indiferencia. El dolo no intencional en la dogmática penal chilena del siglo XXI. Ius et Praxis, 27(1), 190209. Disponible en: http://www.revistaiepraxis.cl/index.php/iepraxis/ article/view/1588

van Weezel, Á. (2020). Una vez más sobre la distinción entre dolo e imprudencia: los casos en que el agente no persigue la realización del tipo penal. Revista de Ciencias Penales, XLVII(2), 43-72. Disponible en: http://revistadecienciaspenales.cl/wp-content/uploads/2020/12/ Revista-CP-Primer-Semestre-2020-corregida-51-79.pdf

van Weezel, Á. (2011). El dolo eventual como espacio de discrecionalidad. Doctrina y Jurisprudencia Penal, 7, 23-52. 